\title{
Reliability and Validity of a New Method for Isometric Back Extensor Strength Evaluation Using A Hand-Held Dynamometer
}

\author{
Hee-won Park, $\mathrm{MD}^{1,2,3}$, Sora Baek, MD, PhD ${ }^{1,2}$, Hong Young Kim, $\mathrm{MS}^{2}$, \\ Jung-Gyoo Park, $\mathrm{MD}^{3}$, Eun Kyoung Kang, $\mathrm{MD}, \mathrm{PhD}^{1,2}$
}

\begin{abstract}
${ }^{1}$ Department of Rehabilitation Medicine, Kangwon National University Hospital, Kangwon National University School of Medicine, Chuncheon; ${ }^{2}$ Center for Farmers' Safety and Health, Kangwon National University Hospital, Chuncheon;

${ }^{3}$ Gangwon-Do Rehabilitation Hospital, Chuncheon, Korea
\end{abstract}

\begin{abstract}
Objective To investigate the reliability and validity of a new method for isometric back extensor strength measurement using a portable dynamometer.

Methods A chair equipped with a small portable dynamometer was designed (Power Track II Commander Muscle Tester). A total of 15 men (mean age, $34.8 \pm 7.5$ years) and 15 women (mean age, 33.1 \pm 5.5 years) with no current back problems or previous history of back surgery were recruited. Subjects were asked to push the back of the chair while seated, and their isometric back extensor strength was measured by the portable dynamometer. Testretest reliability was assessed with intraclass correlation coefficient (ICC). For the validity assessment, isometric back extensor strength of all subjects was measured by a widely used physical performance evaluation instrument, BTE PrimusRS system. The limit of agreement (LoA) from the Bland-Altman plot was evaluated between two methods.

Results The test-retest reliability was excellent (ICC $=0.82 ; 95 \%$ confidence interval, $0.65-0.91$ ). The Bland-Altman plots demonstrated acceptable agreement between the two methods: the lower 95\% LoA was $-63.1 \mathrm{~N}$ and the upper 95\% LoA was $61.1 \mathrm{~N}$.

Conclusion This study shows that isometric back extensor strength measurement using a portable dynamometer has good reliability and validity.
\end{abstract}

Keywords Back muscles, Isometric contraction, Muscle strength dynamometer, Reproducibility of results

Received January 11, 2017; Accepted February 27, 2017

Corresponding author: Sora Baek

Department of Rehabilitation Medicine, Kangwon National University Hospital, Kangwon National University School of Medicine, Baengnyeong-ro 156, Chuncheon 24289, Korea. Tel: +82-33-258-9262, Fax: +82-33-258-9097, E-mail: sora.baek@kangwon.ac.kr

ORCID: Hee-won Park (http://orcid.org/0000-0002-7434-6675); Sora Baek (http://orcid.org/0000-0003-3404-6202); Hong Young Kim (http://orcid. org/0000-0002-4251-9295); Jung-Gyoo Park (http://orcid.org/0000-0001-9970-8321); Eun Kyoung Kang (http://orcid.org/0000-0001-5315-1361).

@ This is an open-access article distributed under the terms of the Creative Commons Attribution Non-Commercial License (http://creativecommons.org/ licenses/by-nc/4.0) which permits unrestricted noncommercial use, distribution, and reproduction in any medium, provided the original work is properly cited. Copyright $\odot 2017$ by Korean Academy of Rehabilitation Medicine 


\section{INTRODUCTION}

Back pain is a common musculoskeletal condition that affects both men and women of different age groups. Due to the multi-factorial nature, it is difficult to identify exact causes of back pain, and treatment is a difficult and time-consuming process. Recently, back rehabilitation programs, which are non-surgical, multi-disciplinary treatments consisting of exercise and physical therapy, have gained popularity for prevention of spinal injury and back pain, and resuming performance of daily activities [1-6].

The key interventions involved in back rehabilitation programs are strengthening and retraining of back muscles [7-10]. Weakness of back muscles has been identified in many back pain cases $[6,7,11]$. Back muscle strength is, therefore, the main target of intervention in back rehabilitation, and evaluation of back muscle strength is crucial $[2,3,6,9]$. Previous studies utilized an isokinetic dynamometer machine for evaluating back muscle strength in the isokinetic or isometric test modes $[6,7,10]$. However, an isokinetic dynamometer machine is neither readily available nor easily movable, and requires sophisticated maintenance efforts and well-trained operators $[7,8,11$ 13].

A portable dynamometer is a small hand-held device that can quantify the muscle strength of extremities in manual muscle testing. The portable dynamometer has a compression force sensor and a control/recording unit. The force sensor is small enough to be mounted on an examiner's hand while resisting against an examinee's action (i.e., hand-held dynamometer). The control/recording unit displays the maximal compression force. To measure the strength using the portable dynamometer, the examiner must use his/her own strength to fully resist the action performed by the subjects. The hand-held dynamometer has not been applied to the measurement of trunk muscle strength because it is difficult for most examiners to resist the examinee's trunk action. The authors have designed a special chair with a seatback that can hold a force sensor unit and resist isometric back extension movement (Fig. 1).

The aim was to assess the reliability of this new method, which combined a portable dynamometer with a chair

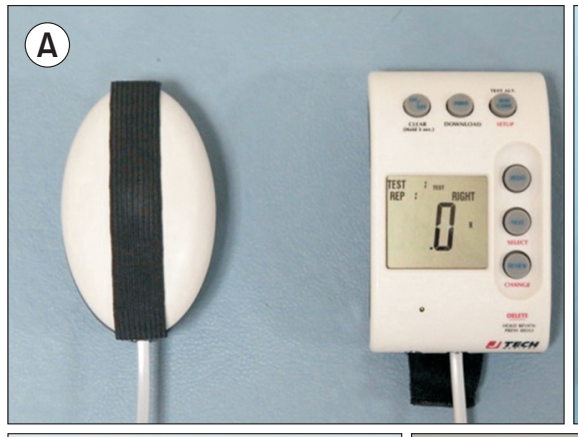

(B)
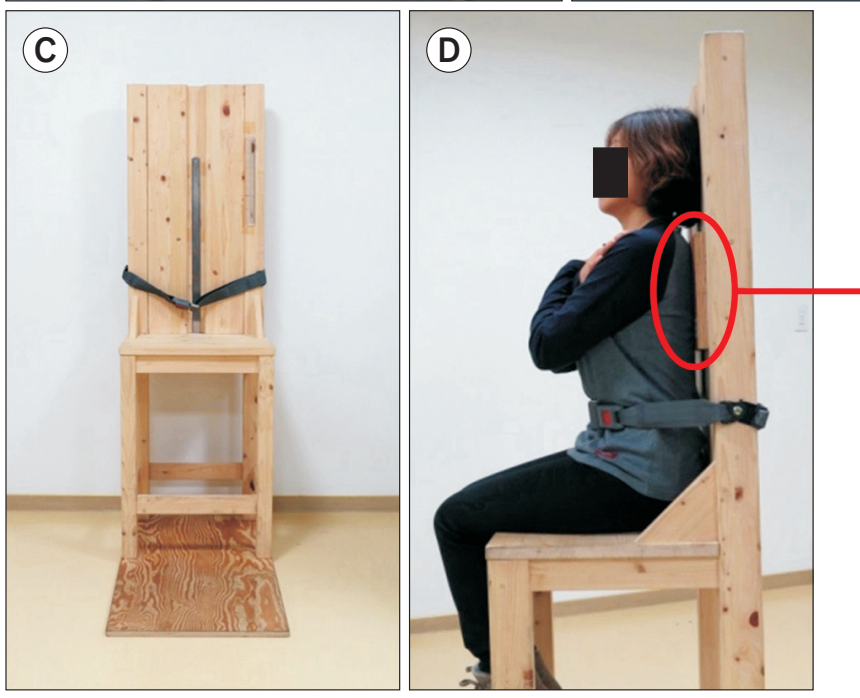

Fig. 1. Device setting for isometric back extensor strength evaluation with portable dynamometer. (A) Portable dynamometer (Power Track II Commander Muscle Tester; JTECH Medical, Midvale, UT, USA) composed of a compression force sensor (left) and a control/ recording unit (right). (B) Square back plate. This wooden plate interface with subjects' back muscle and transmit compression pressure to the attached force sensor unit. (C) Specially designed chair. Vertical linear groove in seatback center is designed for holding a sensor unit with a ruler to set the sensor unit in a specific height. (D) Subject seated in the chair and the pelvis fixed by fastening the seat belt. Square back plate was shown in the red elliptical circle between chair seatback and subjects. The force sensor unit is not visible because it is inserted into the center groove of the chair. 
for isometric back strength evaluation, and compare its strength data with that of the conventional dynamometer system for a validity analysis.

\section{MATERIALS AND METHODS}

The Institutional Review Board of Kangwon National University Hospital approved this study protocol (201401-007-004), and informed consent was obtained from all participants.

\section{Study design}

This was a prospective, comparative, test-retest reliability and validation study. For test-retest reliability analysis, different day values of back strength data measured by portable dynamometer were used. We used a commercially available portable dynamometer (Power Track II Commander Muscle Tester; JTECH Medical, Midvale, UT, USA) originally designed for manual muscle strength testing of extremities. This machine is able to record multiple peak compression forces, and the highest force generated by a subject is recorded automatically at each attempt.

For test-retest reliability, a second measurement was assessed on a different day. On the day of the second measurement, the isometric back extensor strength was also measured using physical performance evaluation instrument, PrimusRS (BTE technologies Inc., Hanover, MD, USA) for the validation study. The PrimusRS is an isokinetic dynamometer machine able to assess isomet- ric, isotonic, and isokinetic muscle forces. The PrimusRS can record accurately up to $1800 \mathrm{in}$. lbs. of isometric force.

\section{Participants}

Fifteen men (mean age, $34.8 \pm 7.5$ years) and 15 women (mean age, 33.1 \pm 5.5 years) aged 19-60 years were included. Subjects were interviewed by a physician and only those who had no current back problems or previous history of back surgery were enrolled.

\section{Device design}

We designed a special chair for measuring isometric back extensor strength using the portable dynamometer (Fig. 1). It is a typical chair with a seat and seatback, but in the center of the seatback, there is a vertical linear hole for holding a sensor unit of the portable dynamometer and a square back plate for stable transmission of pressure generated by the back extensors of a seated subject to the sensor unit. The sensor unit and back plate position is modifiable along the seatback hole to the intended height because a ruler was attached to the seatback. In the lower part of the seat, there is a seat belt for safety and body stabilization. The seat was high enough to lift the feet of subjects off the ground to minimize any force exerted by legs.

\section{Measurement of isometric back extensor strength}

Initially, the subject was seated in the chair and an examiner checked the subject's height of the T7 vertebral
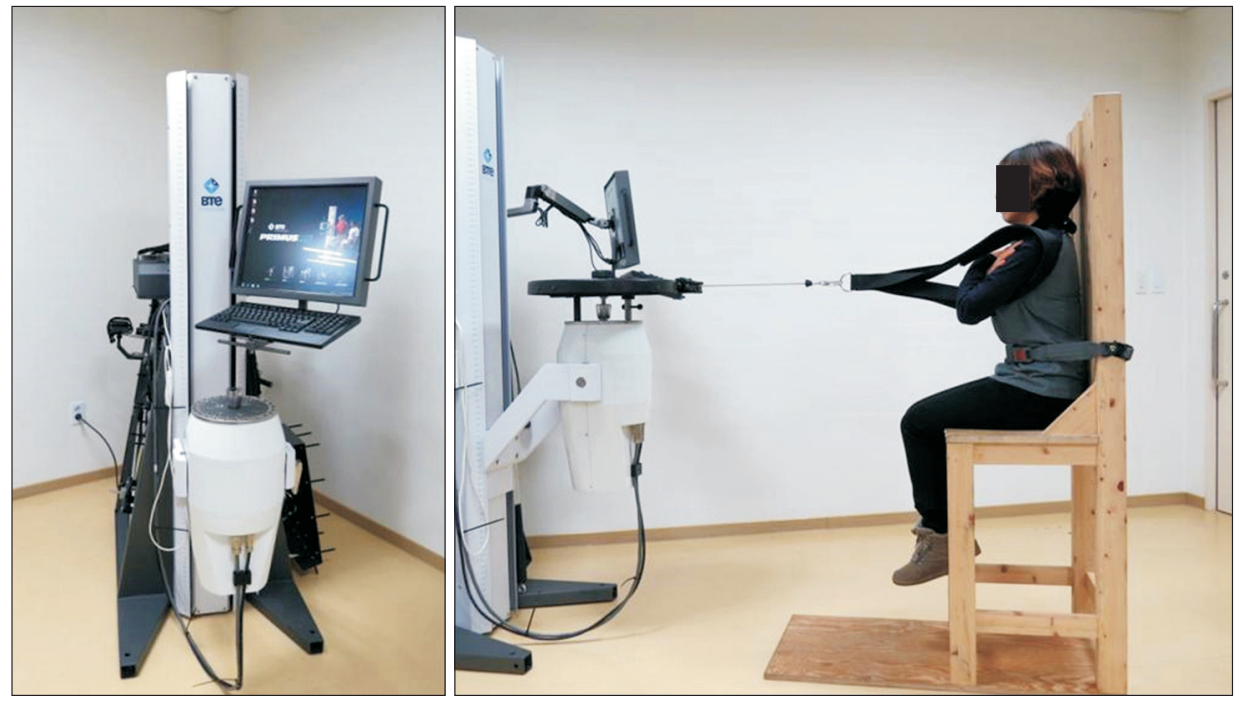

Fig. 2. Isometric back extensor strength evaluation with isokinetic dynamometer machine (PrimusRS; BTE Technologies Inc., Hanover, MD, USA). 
spinous process. Then, the pelvis was fixed by fastening the seat belt. The sensor unit of the portable dynamometer was positioned in the vertical seatback hole with the center of sensor unit at the $\mathrm{T} 7$ spinous process level. The square back plate was positioned between the subject and seatback. The subject placed arms on chest folded and kept shoulders relaxed. With the starting signal, the subject was asked to push the square back plate with his/ her back as far as he/she could for 3 seconds. This process was repeated 5 times, and the mean value was calcu- lated only with 3 median values, excluding the maximum and minimum values. For second measurement, the entire process was repeated for each subject on different days. Finally, two processed values for each participant were acquired for test-retest reliability assessment.

On the same day of the second measurement using the portable dynamometer, the isometric back extensor strength was measured using the PrimusRS system, an isokinetic dynamometer machine (Fig. 2). Between two measurements using the portable dynamometer and Pri-

Table 1. Demographic and isometric back extensor strength data of study subjects

\begin{tabular}{|c|c|c|c|c|c|}
\hline \multirow{2}{*}{ Subject no. } & \multirow{2}{*}{ Age (yr) } & \multirow{2}{*}{ Sex } & \multicolumn{2}{|c|}{ New device (N) } & \multirow{2}{*}{ PrimusRS (N) } \\
\hline & & & Test1 & Test2 & \\
\hline 1 & 32 & F & 247.3 & 224.0 & 190.8 \\
\hline 2 & 29 & M & 259.0 & 259.7 & 268.9 \\
\hline 3 & 42 & M & 248.7 & 233.7 & 229.5 \\
\hline 4 & 33 & M & 155.0 & 182.3 & 227.7 \\
\hline 5 & 57 & M & 211.0 & 232.3 & 233.1 \\
\hline 6 & 41 & $\mathrm{~F}$ & 113.3 & 126.0 & 97.9 \\
\hline 7 & 41 & F & 205.0 & 201.3 & 235.1 \\
\hline 8 & 44 & $\mathrm{~F}$ & 127.7 & 156.3 & 140.8 \\
\hline 9 & 31 & $\mathrm{~F}$ & 219.3 & 239.7 & 191.8 \\
\hline 10 & 32 & M & 165.3 & 139.3 & 181.7 \\
\hline 11 & 27 & M & 198.3 & 189.0 & 250.8 \\
\hline 12 & 27 & M & 146.5 & 178.7 & 210.7 \\
\hline 13 & 30 & M & 226.7 & 211.0 & 277.2 \\
\hline 14 & 34 & M & 220.3 & 176.7 & 238.5 \\
\hline 15 & 27 & $\mathrm{~F}$ & 191.3 & 178.3 & 193.8 \\
\hline 16 & 32 & $\mathrm{~F}$ & 171.7 & 168.7 & 144.5 \\
\hline 17 & 37 & M & 280.3 & 271.0 & 252.9 \\
\hline 18 & 30 & M & 281.7 & 228.7 & 263.4 \\
\hline 19 & 32 & M & 241.7 & 232.7 & 263.4 \\
\hline 20 & 39 & M & 284.7 & 222.7 & 278.6 \\
\hline 21 & 26 & $\mathrm{~F}$ & 186.0 & 167.3 & 176.6 \\
\hline 22 & 34 & F & 160.0 & 132.0 & 237.5 \\
\hline 23 & 29 & $\mathrm{~F}$ & 182.7 & 206.3 & 244.3 \\
\hline 24 & 37 & M & 244.7 & 248.0 & 237.6 \\
\hline 25 & 26 & $\mathrm{~F}$ & 128.3 & 217.7 & 128.7 \\
\hline 26 & 36 & F & 239.0 & 190.0 & 192.6 \\
\hline 27 & 32 & F & 141.3 & 134.7 & 109.7 \\
\hline 28 & 35 & F & 131.3 & 140.3 & 154.0 \\
\hline 29 & 31 & $\mathrm{~F}$ & 247.3 & 276.0 & 261.1 \\
\hline 30 & 36 & M & 292.0 & 313.0 & 264.4 \\
\hline Mean \pm SD & & & $204.9 \pm 52.6$ & $205.9 \pm 49.5$ & $212.6 \pm 51.6$ \\
\hline
\end{tabular}


musRS system, subjects rested for more than 2 hours. The subjects were seated in the same chair described for the portable dynamometer evaluation and wore an evaluation harness anchored to the PrimusRS with a cable. The height of the anchoring cable was modified to the T7 spinous process level with the subjects seated in the chair. They were instructed to maintain an upright posture without leaning on the seatback and shoulders relaxed. With the starting signal, they were asked to push the square back plate with their back as far as they could for 3 seconds with isometric back extension. When subjects did the isometric back extension, the cable anchored to the chest harness was pulled and isometric back extension strength was measured. This process was repeated 5 times, and the mean of 3 median values excluding the maximum and minimum values were calculated. The results were compared with the second measurement data obtained using the portable dynamometer for the validation study.

\section{Statistics}

We calculated the intraclass correlation coefficient (ICC) for assessing test-retest reliability. For validation assessment, we used Pearson correlation coefficient and Bland-Altman plotting and 95\% limits of agreement (95\% LoA) were computed. All statistical analyses were performed using SPSS ver. 20 (IBM, Armonk, NY, USA).

\section{RESULTS}

All demographic data, as well as the mean isometric

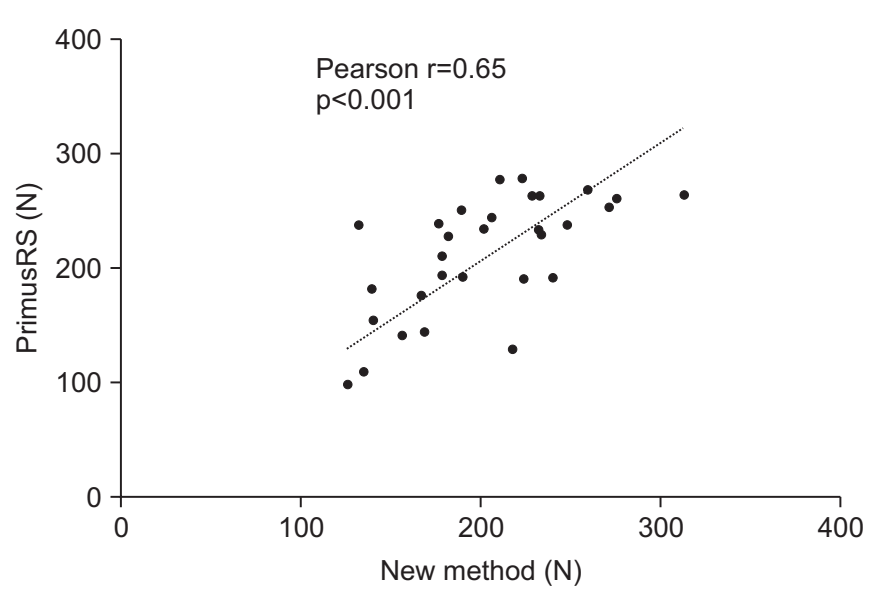

Fig. 3. Correlation between PrimusRS and new method $(\mathrm{p}<0.001)$. back extensor strength values at different test times, are shown in Table 1. The isometric back extensor strength values evaluated by first and second isometric tests using the hand-held dynamometer were $204.9 \pm 52.6 \mathrm{~N}$ and $205.9 \pm 49.5 \mathrm{~N}$, respectively. The isometric strength measured by PrimusRS was 212.6 $\pm 51.6 \mathrm{~N}$.

The test-retest reliability of the measured strength showed good correlation. The ICC of two tests using the hand-held dynamometer was 0.82 (95\% confidence interval, 0.65-0.91) (Table 2).

In the correlation study comparing the PrimusRS system data and our new method, Pearson r was 0.65 (Fig. 3). Bland-Altman plots demonstrated acceptable agreement (Fig. 4) and the 95\% LoA for the PrimusRS method and our new method was $-63.1 \mathrm{~N}$ to $61.1 \mathrm{~N}$.

\section{DISCUSSION}

To assess the test-retest reliability of our new method for isometric back extensor strength evaluation, ICC was utilized. The ICC measures the relative similarity of quantities that share the same observational units of a sampling and/or measurement process [14]. The ICC is considered acceptable at 0.80 or higher. The ICC of our new method demonstrated 0.82 , which indicates noninferior reliability to that of previous studies using the

Table 2. Intraclass correlation coefficient (ICC)

\begin{tabular}{ccc}
\hline ICC & $95 \%$ confidence interval & p-value \\
\hline 0.82 & $0.65-0.91$ & $<0.01$ \\
\hline
\end{tabular}

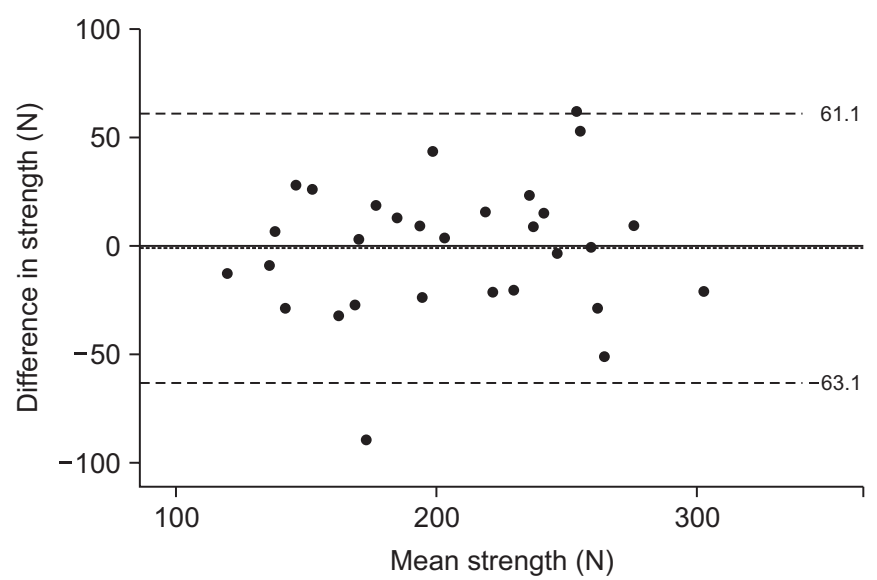

Fig. 4. Bland-Altman plot for muscle strength data. Dotted line indicates $95 \%$ limits of agreement ranging from -63.1 to $61.1 \mathrm{~N}$. 
isokinetic dynamometer machine $[8,12]$.

In validation analysis comparing established and unestablished assessment technology, the Bland-Altman plot is considered the most appropriate method for measuring agreement $[14,15]$. More classical measures such as correlation coefficient (r) and linear regression are inappropriate and often misleading because their primary function is to analyze the strength of the relationship and not the agreement between two variables [15]. In this study, Bland-Altman plots demonstrated that there was an acceptable agreement between the back extensor strength data obtained with the PrimusRS and the data obtained using our new device. The procedure described by Bland and Altman was developed to examine the agreement between two different techniques of quantifying variables, and not necessarily the reliability. The use of the Bland-Altman plot and the limits of agreement as an index of reliability is typically discouraged [14].

Our target structure of strength measurement was the back extensor muscle group. This muscle group mainly consists of multifidus and erector spinae muscles. In muscle fiber type analysis in normal population, the type 1 (slow twitch) muscle fiber was predominant in lumbar multifidus and erector spinae muscles [16], and this may be a reflection of the functional role of the back extensor group as a core stabilizer requiring a high level of endurance. The strength of back extensor muscles decreases in older age as well as female gender, and the rate of decline in strength with age was greater in the aged group and two times higher in women [5]. The incompetence of back extensor muscle is reported to be associated with back pain in both young and old populations $[7,13,17]$. It has been documented that in patients with chronic back pain, the cross-sectional area of the back muscle group on a CT scan was smaller than that of the healthy control group, while the size of the paraspinal muscles was correlated with the isometric back extension strength.

Back muscles protect pain-sensitive structures, such as vertebral columns, intervertebral discs, and facet joints, from excessive stress and loading, therefore the compromising of back muscles may result in injury to these passive structures and induce back pain $[4,10]$. Due to their importance, the strengthening of back muscles may be essential to treat and prevent lower back problems $[2,4]$. In recent years, several systematic reviews have demonstrated that rehabilitative exercise therapy is an effective approach in the treatment of chronic and recurrent back pain [18-20]. Various types of rehabilitation exercises including endurance training, resistive weight training, and stabilization exercises are known to have a beneficial effect in back pain patients, but there is a lack of evidence, as well as a definitive exercise protocol, for most of these exercise types.

We used a seat belt for both pelvic stabilization and hip movement minimization in the sitting position. Hip extensor muscles, such as the glutei and hamstrings, can play a role in back extension which can affect measurement accuracy. To minimize the impact of hip extensors during trunk extension measurement, pelvic stabilization is important $[21,22]$. Another study suggested that combining pelvic stabilization and the semi-sitting position with a hip flexion angle of $45^{\circ}$ would be helpful in increasing the specificity of back extensor exercises [23]. We focused on pelvic stabilization throughout the experiment, however difficulties in device design did not allow for the adaptation of the semi-sitting test position.

Strength testing using the isokinetic dynamometer is the standard quantitative assessment used for evaluation of trunk muscles [24], however the isometric testing method should also be considered for assessing muscle strength $[25,26]$. In isometric muscle contraction, the length of the muscle remains constant and the angular velocity is zero. Almost every isokinetic dynamometer device can be used for isometric testing by simply selecting an angular velocity of zero [24]. Joint angles, however, should be selected carefully in an isokinetic device due to significant force variations throughout the joint's range of motion [24]. It is not clear which of the two, isokinetic or isometric strength measurements, is superior, especially in terms of reliability. Generally, it is believed that isokinetic testing is more responsive to back problems due to the considerable effect of joint movement on pain provocation $[8,17]$. In previous studies on patients with back pain, isokinetic testing produced more varying results in comparison with isometric testing, and repetition of muscle strength evaluation showed significantly increased results, probably due to the 'learning effect' [17]. We demonstrated good reliability of isometric trunk muscle strength assessment using our new device, and the learning effect was negligible.

Another merit of isometric back muscle testing is supported by evidence provided by a long-term outcome re- 
port. A 10-year follow up study on postmenopausal women by Sinaki et al. [26] demonstrated that isometric back strength is closely related to the prevention of vertebral compression fracture. The new isometric testing method discussed here has the added benefits of a simple design and a device that is lightweight, easier to operate and can be transported by one person.

The subjects of this study were healthy volunteers with no current back problems or previous history of back surgery. The reliability could be lower in patients with back pain, although previous study showed comparable reliability of isokinetic measurements between normal and back pain subjects [8]. There is a need for further investigation to assess reliability in the very old population and in those with current back pain.

Compared to the conventional methods, the advantage of the device we designed is high portability and ease of use. This equipment can be transported by one or two people and installed within 5 minutes. It is an ideal evaluation tool for group rehabilitation program or field study outside hospital in the local community.

In conclusion, we developed a simple method for isometric back extensor strength measurement using a specially designed chair and portable hand-held dynamometer. Our method has unmatched simplicity and mobility over the conventional dynamometer-based method, and it demonstrated good reliability and acceptable agreement with measurement data obtained using the isokinetic dynamometer machine in the healthy population.

\section{CONFLICT OF INTEREST}

No potential conflict of interest relevant to this article was reported.

\section{ACKNOWLEDGMENTS}

This study was supported by 2015 Research Grant from Kangwon National University.

\section{REFERENCES}

1. Little P, Smith L, Cantrell T, Chapman J, Langridge J, Pickering R. General practitioners' management of acute back pain: a survey of reported practice compared with clinical guidelines. BMJ 1996;312:485-8.
2. Chok B, Lee R, Latimer J, Tan SB. Endurance training of the trunk extensor muscles in people with subacute low back pain. Phys Ther 1999;79:1032-42.

3. Kaser L, Mannion AF, Rhyner A, Weber E, Dvorak J, Muntener M. Active therapy for chronic low back pain: part 2. Effects on paraspinal muscle crosssectional area, fiber type size, and distribution. Spine (Phila Pa 1976) 2001;26:909-19.

4. Moon HJ, Choi KH, Kim DH, Kim HJ, Cho YK, Lee KH, et al. Effect of lumbar stabilization and dynamic lumbar strengthening exercises in patients with chronic low back pain. Ann Rehabil Med 2013;37:110-7.

5. Singh DK, Bailey M, Lee R. Decline in lumbar extensor muscle strength the older adults: correlation with age, gender and spine morphology. BMC Musculoskelet Disord 2013;14:215.

6. Cho KH, Beom JW, Lee TS, Lim JH, Lee TH, Yuk JH. Trunk muscles strength as a risk factor for nonspecific low back pain: a pilot study. Ann Rehabil Med 2014; 38:234-40.

7. Bayramoglu M, Akman MN, Kilinc S, Cetin N, Yavuz $\mathrm{N}$, Ozker R. Isokinetic measurement of trunk muscle strength in women with chronic low-back pain. Am J Phys Med Rehabil 2001;80:650-5.

8. Keller A, Hellesnes J, Brox JI. Reliability of the isokinetic trunk extensor test, Biering-Sørensen test, and Astrand bicycle test: assessment of intraclass correlation coefficient and critical difference in patients with chronic low back pain and healthy individuals. Spine (Phila Pa 1976) 2001;26:771-7.

9. Hanada EY, Hubley-Kozey CL, McKeon MD, Gordon SA. The feasibility of measuring the activation of the trunk muscles in healthy older adults during trunk stability exercises. BMC Geriatr 2008;8:33.

10. Lee HJ, Lim WH, Park JW, Kwon BS, Ryu KH, Lee JH, et al. The relationship between cross sectional area and strength of back muscles in patients with chronic low back pain. Ann Rehabil Med 2012;36:173-81.

11. Bernard JC, Boudokhane S, Pujol A, Chaleat-Valayer E, Le Blay G, Deceuninck J. Isokinetic trunk muscle performance in pre-teens and teens with and without back pain. Ann Phys Rehabil Med 2014;57:38-54.

12. Hutten MM, Hermens HJ. Reliability of lumbar dynamometry measurements in patients with chronic low back pain with test-retest measurements on different days. Eur Spine J 1997;6:54-62. 
13. Yahia A, Ghroubi S, Kharrat O, Jribi S, Elleuch M, Elleuch MH. A study of isokinetic trunk and knee muscle strength in patients with chronic sciatica. Ann Phys Rehabil Med 2010;53:239-44, 244-9.

14. Weir JP. Quantifying test-retest reliability using the intraclass correlation coefficient and the SEM. J Strength Cond Res 2005;19:231-40.

15. Bland JM, Altman DG. Statistical methods for assessing agreement between two methods of clinical measurement. Lancet 1986;1:307-10.

16. Rantanen J, Rissanen A, Kalimo H. Lumbar muscle fiber size and type distribution in normal subjects. Eur Spine J 1994;3:331-5.

17. Gruther W, Wick F, Paul B, Leitner C, Posch M, Matzner M, et al. Diagnostic accuracy and reliability of muscle strength and endurance measurements in patients with chronic low back pain. J Rehabil Med 2009;41:613-9.

18. Pengel HM, Maher CG, Refshauge KM. Systematic review of conservative interventions for subacute low back pain. Clin Rehabil 2002;16:811-20.

19. Mayer J, Mooney V, Dagenais S. Evidence-informed management of chronic low back pain with lumbar extensor strengthening exercises. Spine J 2008;8:96113.

20. Scharrer M, Ebenbichler G, Pieber K, Crevenna R, Gruther W, Zorn C, et al. A systematic review on the effectiveness of medical training therapy for subacute and chronic low back pain. Eur J Phys Rehabil Med 2012;48:361-70.

21. Graves JE, Webb DC, Pollock ML, Matkozich J, Leggett $\mathrm{SH}$, Carpenter DM, et al. Pelvic stabilization during resistance training: its effect on the development of lumbar extension strength. Arch Phys Med Rehabil 1994;75:210-5.

22. da Silva RA, Lariviere C, Arsenault AB, Nadeau S, Plamondon A. Pelvic stabilization and semisitting position increase the specificity of back exercises. Med Sci Sports Exerc 2009;41:435-43.

23. da Silva RA, Lariviere C, Arsenault AB, Nadeau S, Plamondon A. Effect of pelvic stabilization and hip position on trunk extensor activity during back extension exercises on a Roman chair. J Rehabil Med 2009;41: 136-42.

24. Maud PJ, Foster C. Physiological assessment of human fitness. 2nd ed. Champaign, IL: Human Kinetics; 2006. p. 131-8.

25. Limburg PJ, Sinaki M, Rogers JW, Caskey PE, Pierskalla BK. A useful technique for measurement of back strength in osteoporotic and elderly patients. Mayo Clin Proc 1991;66:39-44.

26. Sinaki M, Khosla S, Limburg PJ, Rogers JW, Murtaugh PA. Muscle strength in osteoporotic versus normal women. Osteoporos Int 1993;3:8-12. 